\title{
¿Los alumnos de las escuelas \\ privadas están mejor preparados \\ para ingresar a la universidad?
}

\section{Artículo de investigación}

Revista Colombiana de Educación, N. ${ }^{\circ} 70$. Primer semestre de 2016 Bogotá, Colombia.

\section{//Are Private School Students Better \\ Trained for University Admission?}

\section{//Os alunos das escolas privadas \\ estão melhor preparados para o vestibular universitário?}

Tamara Vinacur*

\begin{abstract}
Candidata a doctora en Educación de la Universidad de San Andrés, Argentina, magíster en Medición, Estadísticas y Evaluación de la Universidad de Columbia y licenciada en Ciencias de la Educación de la Universidad de Buenos Aires. Consultora en la Organización de Estados Iberoamericanos. Buenos Aires, Argentina. Correo electrónico: tvinacur@gmail.com
\end{abstract}

\section{Resumen}

El ingreso a la educación superior refleja oportunidades diferenciales de acceso, permanencia y logro, incluso en instituciones de educación superior universitaria con ingreso irrestricto. Este estudio indaga la incidencia del carácter estatal o privado del colegio al que asistieron los alumnos en la secundaria en su desempeño académico en el primer año en la Universidad de Buenos Aires (UBA). Se consideran algunas variables personales y familiares de los alumnos ingresantes y características del radio censal donde está ubicado el establecimiento de nivel secundario al que asistieron. Se trabaja con una muestra de 8542 estudiantes que ingresaron en el año 2010 al Ciclo Básico Común de la UBA, que ya cursaron Introducción al Estudio de la Sociedad y el Estado, una de las dos materias comunes a todas las carreras. El estudio identifica que los alumnos más jóvenes (17-19 años) tienen en promedio mejor rendimiento, con independencia del radio censal y el sector de gestión de la escuela secundaria a la que asistieron. También se encuentra que los alumnos provenientes de escuelas secundarias privadas (en particular aquellas ubicadas en zonas de mayor poder adquisitivo) contaron con mayores probabilidades de transitar exitosamente el primer año de universidad, según se evidencia tanto en la calificación obtenida como en el tiempo requerido para lograrlo. Sin embargo, se identifica un comportamiento diferencial entre quienes asistieron a escuelas estatales ubicadas en entornos con alto nivel de pobreza estructural, donde esta diferencia entre sectores de gestión se diluye. Finalmente, se advierte sobre la necesidad de considerar las características de los alumnos y sus hogares como posibles condicionantes para el reclutamiento.

\section{Abstract}

Admission to higher education reflects a differential access to educational opportunities, continuity and achievement, even in institutions without any entry exams or requirements. The article studies the impact of public and private secondary schools in the academic achievement of freshmen students at the Universidad de Buenos Aires (UBA). Some student's personal and family-related variables as well as characteristics of the census radio where the secondary schools are located are taken into consideration. The sample consists of 8542 students who enrolled to the Common Basic Cycle of the UBA in 2010 and took the subject "Introduction to the Study of Society and State" (one of the two subjects common to all majors.) The analysis shows that, as a rule, younger students (17-19 years

\section{Palabras clave}

Universidad, escuela secundaria, educación privada, ingreso, logro académico

\section{Keywords}

University, secondary school, private education, admission, academic success

\section{Palavras chave}

Universidade, escola secundaria, educação particular, vestibular, formatura acadêmica. 
old) perform better academically, no matter the influence of the census radio and the type of the secondary school (private or public) they attended to. It is also identified that students from private secondary schools - mainly those from schools in high-income areas - are more likely to have a strong academic performance as freshmen, as evidenced in their final grade as well as in the time needed to get it. However, it is found that this difference disappears among those who attended public schools located in poorer areas, when compared to those who attended private schools in similar areas. Finally, attention is drawn to the need to consider the characteristics of students and their homes as possible recruitment requirements.

\section{Resumo}

O vestibular à educação superior reflete oportunidades diferenciais de acesso, permanência e formatura, mesmo em instituições de educação superior universitário com ingresso sem restrições. Neste estudo pesquisa a incidência do caráter estatal ou privado do colégio ao que assistiram os estudantes no ensino secundário, em seu desempenho acadêmico no primeiro ano na "Universidad de Buenos Aires" (UBA). Consideram-se algumas variáveis pessoais e familiares dos alunos ingressantes, e caraterísticas do rádio do censo onde está localizado o estabelecimento do nível secundário ao que assistiram. Trabalha-se com uma mostra de 8542 estudantes que ingressaram no ano 2010 ao Ciclo Básico Comum da UBA, que já cursaram "Introducción al Estudio de la Sociedad y el Estado", uma das duas matérias comuns a todas os cursos universitários. O estudo identifica que os alunos mais jovens (17-19 anos) têm em média melhor rendimento, com independência do rádio do censo e o setor de gestão da escola secundária à que assistiram. Também achamos que os alunos provenientes de escolas secundárias particulares (em especial aquelas localizadas em zonas de maior poder aquisitivo), tiveram maiores probabilidades de percorrer com sucesso o primeiro ano de universidade, segundo se evidência tanto na qualificação obtida quanto no tempo requerido para logra-lo. Embora, identifica-se um comportamento diferencial entre quem assistiu a escolas estatais localizadas em contextos com alto nível de pobreza estrutural, onde esta diferença entre setores de gestão se dilui. Finalmente, se faz um chamado sob a necessidade de considerar as caraterísticas dos alunos e seus lares como possíveis condicionais para o recrutamento.

El Ciclo Básico Común (СвC) de la Universidad de Buenos Aires (UBA, Argentina) ${ }^{1}$ se inicia en 1985 y constituye el primer ciclo de los estudios universitarios que es común a las distintas facultades. Dado que se trata de una modalidad de ingreso libre e irrestricto, el CBC se constituye en el primer año universitario destinado fundamentalmente a la orientación y nivelación de los alumnos que ingresan. Para ello, el plan de estudios prevé una formación

1 La Universidad de Buenos Aires es una universidad pública argentina, fundada en 1821 Actualmente es una de las diez universidades latinoamericanas que integran la clasificación de Shangai 2014 (se ubica en segundo lugar después de la Universidad de San Pablo). Al igual que todas las universidades argentinas es gratuita (su financiamiento depende del Estado argentino), autónoma (posee su propio sistema de gobierno), libre (hay libertad de cátedra) y laica, de acuerdo a lo establecido a partir de la Reforma Universitaria de 1918. 
básica integral e interdisciplinaria a partir de seis materias obligatorias: dos comunes para todas las carreras (Introducción al Pensamiento Científico e Introducción al Estudio de la Sociedad y el Estado), dos que varían según la orientación en la que está comprendida la carrera elegida y las dos últimas que son específicas de cada carrera.

En un contexto de incremento en la matrícula universitaria y de gran diversidad en el perfil de los alumnos que ingresan (Trow, 2005; Unesco, 2002), resulta necesario conocer el modo en que la experiencia educativa anterior afecta los resultados obtenidos en el primer año. En este sentido, el desempeño académico en el primer año de la universidad condensa en cierto modo gran parte de la experiencia escolar previa (en este caso la educación secundaria), pero también refleja aquella ocurrida en años anteriores, que arrastra los problemas de aprendizaje y de socialización de un nivel a otro (Francis et al., 1994; García de Fanelli, 2005; Porcel et al., 2010; Williamsom, et al., 1991 en García Jiménez et al., 2000).

En ese marco, este estudio analiza si el sector de gestión, público o privado, de la escuela a la que asistieron los estudiantes en la secundaria influye en su posterior logro académico. Para ello se considera la calificación obtenida y el tiempo en que logran aprobar la materia número 24: Introducción al Estudio de la Sociedad y el Estado (en adelante, Sociedad y Estado). Para ello se trabaja con una muestra de 8542 estudiantes ${ }^{2}$ que ingresaron en un mismo periodo (ciclo 2010) y cursaron la referida materia.

La materia analiza desde una perspectiva histórica los distintos procesos históricos que atravesaron a la Argentina a lo largo del siglo xx y tiene una intensidad horaria de cuatro horas semanales, distribuidas en dos jornadas. En el año 2010 se dictaba esta materia en dieciocho cátedras.

\section{La experiencia educativa anterior y el logro en la universidad}

La literatura internacional identifica que son diversos los factores que afectan el logro de los alumnos en educación superior. Se señalan aspectos individuales (como edad, género, estado civil, integración social y antecedentes); aspectos académicos (factores de la experiencia educativa previa, como algunas características del programa elegido: orientación profesional, rendimiento académico, satisfacción con el programa y calidad de este); aspectos institucionales (normativa académica, becas y financiamiento, recursos universitarios, relaciones con profesores, entre otros); y aspectos socioeconómicos (estrato socioeconómico del estudiante, situación laboral del estudiante y de sus padres, nivel educativo

2 La información analizada proviene de una encuesta aplicada a quienes ingresaron, durante su inscripción al CBC. Se consideran como objeto de este estudio aquellos admitidos (cohorte 2010) que asistieron a instituciones educativas de nivel secundario en el ámbito de la Ciudad Autónoma de Buenos Aires. 
de los padres, entre otros). En algunos casos, se consideran los aspectos socioeconómicos como parte de los factores del hogar de origen, entendiendo por ello los valores, recursos materiales, estrategias de reproducción biológica, económica y social (Brunner \& Elacqua, 2004; Ladrón de Guevara, 2010).

Se reconoce que es en el primer año cuando se observan mayores tasas de deserción (Crissman \& Upcraft, 2005; Tinto, 2001) y que las dificultades académicas que se observan actúan en convergencia con otros obstáculos de carácter estructural que afectan el desempeño y pueden desencadenar el abandono (Amago, 2004). Así, la preparación insuficiente para el ingreso en educación superior se asocia con el estatus socioeconómico de los alumnos ingresantes, lo que refleja una profunda desigualdad entre distintos segmentos sociales. En ese sentido, se sugiere que "[...] la preparación académica en el punto de partida es un factor decisivo de la persistencia y la deserción [...] conforma el condicionante prevalente, el más eficaz para la conclusión del grado [...]" (Ezcurra, 2011, p. 37).

Distintas investigaciones (Jansen \& Suhre, 2010; McKenzie \& Schweitzer, 2001) señalan que la formación previa tiene un impacto en el desempeño académico de los alumnos en la universidad. Se hace referencia a una "desigualdad cultural socialmente condicionada" como reflejo de la segmentación de la oferta educativa según la condición social (Ezcurra, 2009), que afecta particularmente a los alumnos de estatus socioeconómico en desventaja (Boulet, 2005; Gessaghi \& Llinás, 2005; Plotno, 2009). De este modo, las posibilidades de acceder a la enseñanza superior son el resultado de una selección que se ejerce a lo largo de todo el recorrido educativo de las personas y que tiene un rigor muy desigual según el origen social (Bourdieu \& Passeron, 1964) ${ }^{3}$.

Así, la calidad de la educación secundaria se constituye como un factor que afecta el acceso a oportunidades y a la movilidad social (Correa, 2004). Algunos de los aspectos que diferencian la formación recibida en el nivel secundario se expresan en dificultades de comprensión lectora, metodología de estudio, producción escrita y resolución de problemas matemáticos (Camilloni, 2009; García de Fanelli, 2005). Algunos autores identifican distintos tipos de capacidades requeridas para estudiar y aprender en la universi-

3 En el caso de la escuela secundaria, se reconoce que en Argentina existe una segmentación del sistema educativo que da lugar a circuitos educativos diferenciados de distinta calidad según la población, lo que refleja la reproducción de las diferencias de origen socioeconómico (Filmus, Miranda \& Otero, 2004). 
dad, particularmente vinculadas con la comprensión de textos y escritura académica (Alava 1999; Cols, 2005 y 2007; Coulon, 1997; Entwistle, 2000; Ezcurra, A. M. 2003 y 2005; Rinaudo, et al., 2003; Tinto, 2001). En primer lugar, se hace referencia a la comprensión y el manejo de herramientas conceptuales de orden disciplinar. En segundo lugar, al dominio de capacidades generales del pensamiento o de orden cognitivo, tales como búsqueda, selección, agrupamiento y procesamiento de información, análisis y solución de problemas, argumentación, entre otras. Al respecto, Teobaldo (2002) encuentra diferencias sustantivas entre los alumnos ingresantes en términos de su capacidad analítica, resolución de problemas, conocimientos de matemática y capacidad de redactar un texto con coherencia. Finalmente, se señala la necesidad de contar con capacidades básicas para el trabajo académico ligadas a la condición de estudiante universitario, tales como organización del tiempo, sistematicidad y disciplina de estudio, habilidades para la intervención en clase, desempeño en grupos de trabajo y presentación de trabajos (Coulon, 1997; Sirota, 2000; Wingate, 2007).

Es posible advertir evidencias contrapuestas con relación a la influencia que ejerce el tipo de establecimiento secundario al que asistieron los alumnos. Por un lado, algunos autores (Ponsot et al. 20094; Porcel et al.,

4 Analiza los ingresantes entre 2003-2007 a la Facultad de Ciencias Económicas y Sociales (FACES) de la Universidad de Los Andes (ULA), Venezuela.
$2010^{5}$ ) no encuentran diferencias significativas en función del sector de gestión del que provienen los alumnos (establecimientos educativos estatales o privados). Sin embargo, hallazgos de otras investigaciones indican que las características del colegio de procedencia se encuentran asociadas al rendimiento académico universitario (Birch \& Miller, 2007; Di Gresia et al., 20026'; McDonough, 1997; Valdivieso et al., 2004). En el análisis del ingreso a la licenciatura de la Universidad Nacional Autónoma de México (UNAm), Guzmán Gómez y Serrano Sánchez (2011, p. 43) encontraron que

[...] una vez controladas
las demás características
de los jóvenes, el efecto
mayor se aprecia cuando
éstos realizaron la educa-
ción básica y media supe-
rior en escuelas privadas,
ya que rebasa en $50 \%$ la
frecuencia de los alumnos
aceptados comparados
con los que provienen de
escuelas públicas.

Las autoras lo explican a partir de dos hipótesis: la primera hace referencia a una supuesta ventaja comparativa de la escuela privada con

5 Realiza el análisis con alumnos ingresantes a la Facultad de Ciencias Exactas y Naturales y Agrimensura de la Universidad Nacional del Nordeste en Corrientes, Argentina.

6 Di Grescia (2002, p.13) analiza los datos de Censo de Estudiantes de Universidades Nacionales de 1994, en el que se relevaron datos de todos los alumnos inscritos en carreras de grado en universidades públicas de la Argentina y señala que los alumnos de las escuelas privadas obtienen mejores calificaciones. 
relación a los factores internos (tipo de enseñanza, condiciones de enseñanza, mejor infraestructura, grupos más pequeños, personal mejor calificado), lo cual podría expresarse en un mejor nivel académico. La segunda hipótesis remite a los factores externos de la escuela, esto es, el capital cultural y económico de los alumnos de las escuelas privadas, que cuentan con un mayor acceso a recursos educativos y culturales, y con padres que alcanzaron un mayor nivel de instrucción.

Este trabajo se focaliza principalmente en explorar el modo en que el sector de gestión de la escuela secundaria a la que asistieron los estudiantes puede haber favorecido, o desalentado, una mejor formación inicial para lograr una calificación más alta en la materia seleccionada o su aprobación en el tiempo requerido. Se tomarán en cuenta en el análisis la influencia de las características del entorno en que se encuentra emplazado el establecimiento educativo de nivel secundario, así como algunas características personales de los alumnos y sus familias.

\section{Metodología}

El análisis se realiza sobre datos secundarios cuantitativos. Se decidió trabajar con los datos de los alumnos ingresantes en el ciclo 2010 dado que no se cuenta con información sistematizada para años anteriores acerca de los establecimientos educativos donde los alumnos realizaron el nivel secundario ${ }^{7}$.

Las bases de datos se construyen a partir de la información proporcionada tanto por el CBC como por la Dirección Nacional de Información y Evaluación de la Calidad Educativa (DiNiece) del Ministerio de Educación de la Nación. La información proporcionada por este organismo se vincula a través de la Clave Única de Establecimientos (CUE) con las bases de información sociodemográfica de los alumnos (encuesta de preinscripción) e información académica, provenientes del CBC.

\section{Universo estudiado}

El universo se encuentra definido a partir de los siguientes criterios: se trata de alumnos que efectivamente cursaron el СвC en el 2010 como alumnos regulares, que cursaron la materia Sociedad y Estado (24) y que asistieron a la escuela secundaria en la Ciudad de Buenos Aires (СВA), la que pudo haber sido identificada mediante

7 Antes del 2010 se formulaba de una pregunta abierta, lo cual requiere de la carga del personal del CBC. 
su CUE en el Padrón de establecimientos 2013 (actualizado al 4 de marzo de 2013), que figura en la encuesta de preinscripción utilizada por el СВC para relevar información demográfica de los alumnos ingresantes ${ }^{8}$. El total de sujetos comprendidos en la muestra es de 8542 estudiantes, de los cuales el $42 \%$ asistió a escuelas públicas y el $58 \%$ a escuelas privadas.

\section{Estrategia metodológica}

Se analiza la calificación obtenida por los alumnos como nota final del curso y el tiempo transcurrido hasta la aprobación de la materia, en función de las características del establecimiento educativo de nivel secundario al que asistieron. Se consideran las características de nivel socioeconómico correspondientes al radio censal donde se encuentra ubicada la escuela y el sector de gestión. La consideración del radio censal en el análisis permite, en cierta medida, controlar la heterogeneidad de las escuelas, principalmente aquellas del sector de gestión privada. Entre estas existen algunas "de élite" con cuotas sumamente elevadas y otras como las confesionales, que cuentan

8 En el proceso de acondicionamiento de la base de datos se tomó la decisión de eliminar los registros (alumnos) para los que no se contaba con suficiente información de alguna de las variables seleccionadas. En relación con la identificación de los colegios secundarios, se han realizado análisis de consistencia para garantizar que no se han excluido establecimientos que presenten características específicas que pudieran cuestionar la validez del estudio. con un alto porcentaje de aporte estatal $^{9}$. Se realizan análisis descriptivos multivariados, considerando a la vez variables personales y familiares de los estudiantes.

Las variables utilizadas en el análisis son algunas relativas a las características de los estudiantes (género, edad, nacionalidad, hijos, estado civil), de sus hogares (nivel educativo de la madre y del padre, servicio de salud donde concurre para realizar una consulta médica ${ }^{10}$ ) $y$ finalmente, otras relativas al establecimiento educativo donde realizaron sus estudios secundarios (sector de gestión, NBI Necesidades Básicas Insatisfechas, del radio censal donde está ubicado el colegio (nivel_NBI).

La variable nivel_NBI se construyó a partir de la información sobre el porcentaje de hogares en situación de pobreza estructural en cada uno de los radios censales en que se organiza la СBA para llevar adelante distintos relevamientos oficiales de información. Para ello se consideró

9 Argentina cuenta con un sistema de subsidios estatales a la educación privada desde el año 1947, financiando parte o la totalidad de los salarios de los docentes de las escuelas privadas a las que asistiera la población de menores ingresos (Morduchowicz, 1999)

$10 \mathrm{El}$ acceso al sistema de salud se utiliza como proxy del nivel socioeconómico del hogar del alumno, considerando que en Argentina los sectores más vulnerables tienen como única asistencia de salud los hospitales públicos, mientras que los sectores formalizados cuentan con obras sociales sindicales, y los sectores más favorecidos (profesionales y autónomos) utilizan la medicina prepaga o privada (Acuña \& Chudnovsky, 2002). 
el $\mathrm{NBI}^{11}$ correspondiente al radio censal donde está ubicado cada establecimiento educativo de nivel secundario donde asistieron los alumnos del СвС. Se construyeron cuatro categorías teóricas en función de la incidencia de NBI en cada radio censal, y los asentamientos, contemplando los siguientes niveles socioeconómicos: incidencia de NBI baja, incidencia de NBI media, incidencia de NBI alta y asentamientos ${ }^{12}$.

\section{Resultados}

El análisis contempla, en primer lugar, las características de los alumnos ingresantes en el 2010. Se consideran las diferencias existentes entre el perfil de alumnos ingresantes que realizaron sus estudios secundarios en establecimientos de gestión estatal o privada, y a su vez el porcentaje de NBI del radio censal donde está ubicada la escuela secundaria a la que asistieron.

\section{Características de los alumnos ingresantes}

En relación a las características demográficas de los ingresantes al СвC en el año 2010, se observa que el mayor porcentaje son mujeres procedentes de escuelas ubicadas en entornos de baja pobreza estructural, siendo la participación de este segmento levemente superior en el caso de las que asistieron a secundarias privadas ( $45 \%$, frente al $41 \%$ de las que asistieron a secundarias estatales) (véase el cuadro 4 en el anexo).

11 Se consideran hogares con NBI aquellos en los cuales está presente al menos uno de los siguientes indicadores de privación: "Hogares que habitan viviendas con más de 3 personas por cuarto (hacinamiento crítico). Hogares que habitan en una vivienda de tipo inconveniente (pieza de inquilinato, vivienda precaria u otro tipo). Hogares que habitan en viviendas que no tienen retrete o tienen retrete sin descarga de agua. Hogares que tienen algún niño en edad escolar que no asiste a la escuela. Hogares que tienen 4 o más personas por miembro ocupado y en los cuales el jefe tiene bajo nivel de educación (sólo asistió dos años o menos al nivel primario)". Fuente: Indec, Situación y evolución social (Síntesis N. ${ }^{\circ}$ )

12 Villa (de emergencia) o asentamiento: núcleo habitacional ubicado en terrenos fiscales o de terceros que fueron ocupados en forma ilegal. En las villas de emergencia, las viviendas no están ubicadas con criterio de ordenamiento edilicio, sino que aparecen distribuidas en el terreno, sin separaciones entre ellas, no hay trazado de calles de circulación ni subdivisión ordenada de terrenos. Es decir no son barrios amanzanados, sino organizados a partir de intrincados pasillos, donde por lo general no pueden pasar vehículos, producto de ocupaciones lentas y no planificadas de tierras urbanas y suburbanas de muy baja calidad. Se incluyen entre ellas, las villas que se encuentran en proceso de urbanización. Para los fines de este trabajo, la denominación comprende los asentamientos, villas de emergencia y núcleos habitacionales transitorios, donde se registra un muy alto nivel de NBI (http://Www.indec.gov.ar/censo2010/consOLIDADO\%20 PARA \% 20CONSEJO \%20y\%20PRENSA-CNPV\%202010\%20y\%20EXPERIMENTAL.pdf). 
En cuanto a la edad de los alumnos ingresantes, la mayoría tiene hasta 19 años y asistió a escuelas ubicadas en zonas con bajo nivel de pobreza, aunque la combinación de estos atributos se verifica en el $67 \%$ de los que concurrieron a secundarias privadas y en el $49 \%$ de los alumnos que concurrieron a secundarias estatales.

Por su parte, el mayor porcentaje de ingresantes no tiene hijos y asistió a escuelas localizadas en entornos favorables. Esta situación representa a un $73 \%$ de los alumnos procedentes de secundarias privadas y a un $65 \%$ de aquellos que cursaron estudios en establecimientos de gestión estatal.

Con respecto a la nacionalidad de los alumnos del СBC, la mayoría son argentinos y estudiaron en escuelas ubicadas en contextos favorables. Sin embargo, esta situación se verifica en el $73 \%$ de los alumnos que asistieron a secundarias privadas y en el $65 \%$ de los que fueron a secundarias estatales.

Considerando exclusivamente al segmento de alumnos que asistieron a escuelas ubicada en entornos de baja pobreza estructural, se observa que el porcentaje de ingresantes procedentes de secundarias privadas con padres que completaron estudios universitarios duplica al porcentaje de alumnos procedentes de secundarias estatales con padres universitarios ( $31 \%$ y $15 \%$ respectivamente). La misma situación se verifica en el caso de las madres. Aun así, las incidencias más elevadas se verifican entre los ingresantes al СвС procedentes de escuelas ubicadas en entornos favorables cuyos padres no poseen estudios superiores.

Se observa finalmente que el $25 \%$ de los ingresantes al СвС que proceden de secundarias privadas ubicadas en zonas favorables cuentan con servicios de medicina prepaga o concurren a hospitales privados antes un problema de salud. Esta incidencia se reduce al $12 \%$ en el caso de los ingresantes que proceden de secundarias estatales. Sin embargo, los porcentajes más elevados se verifican entre los ingresantes que asistieron a escuelas localizadas en entornos favorables, ya sea del sector privado o estatal, que disponen de obra social (sistema de acceso a la salud asociado al empleo formal propio o de los padres).

En síntesis, entre los ingresantes al СвC que cursaron sus estudios secundarios en escuelas privadas ubicadas en entornos favorables, la incidencia de las mujeres, de los jóvenes cuya edad refleja el recorrido de una trayectoria escolar sin demoras, de aquellos que no tienen hijos, de los que tienen padres y madres con estudios universitarios completos, y de los que acceden a servicios privados de salud, supera en cada caso al porcentaje de los ingresantes que asistieron a secundarias estatales.

\section{Logro académico y escuela secundaria de origen}

En primer lugar, en el cuadro 1 se analiza la incidencia del nivel socioeconómico del radio censal para cada 
sector de gestión de la escuela secundaria a la que asistieron los alumnos. Esta apertura permite considerar no solo las diferencias entre las escuelas de gestión estatal y privada, sino que se incorpora la variable que categoriza el nivel socioeconómico del espacio urbano (radio censal) en el cual se halla ubicada la escuela secundaria a la que asistieron. Se asume que esta variable es un proxy de la base de reclutamiento de los jóvenes que asisten a cada establecimiento educativo.

Se observa que hay una mayor proporción de ingresantes provenientes de escuelas ubicadas en radios censales con bajo nivel de pobreza estructural, tanto de escuelas estatales como del sector privado, lo cual da cuenta de una composición de alumnos provenientes de sectores de mayores ingresos. También se advierte una proporción de alumnos que asistieron a establecimientos de gestión privada ubicados en radios censales de alto nivel de pobreza estructural. Al respecto, cabe aclarar que el Estado otorga ayuda financiera a muchas instituciones privadas, lo cual permite abaratar los precios de matrícula ${ }^{13}$, particularmente en el caso de muchas escuelas parroquiales (Narodowski \& Andrada, 2000). Esto facilita el acceso a los alumnos de menores recursos (artículo 25 de la Constitución de la Ciudad de Buenos Aires, artículo 65 de la Ley de Educación Nacional N. 26.206).

Cuadro 1. Ingresantes al CBC por del nivel socioeconómico del radio censal, según sector de gestión del colegio secundario al que asistieron los alumnos

\begin{tabular}{|l|cc|cc|}
\hline Sector de gestión & \multicolumn{2}{|c|}{ Estatal } & \multicolumn{2}{c|}{ Privada } \\
\hline NIVEL_NBI & Frecuencia & Porcentaje & Frecuencia & Porcentaje \\
\hline NBI bajo & 2476 & 69 & 3678 & 74 \\
\hline NBI medio & 527 & 15 & 1000 & 20 \\
\hline NBI alto & 456 & 13 & 178 & 4 \\
\hline Asentamientos & 109 & 3 & 118 & 2 \\
\hline Total & 3568 & $100 \%$ & 4974 & $100 \%$ \\
\hline
\end{tabular}

Fuente: Elaboración propia con base en información proporcionada por el Departamento de Alumnos del Ciclo Básico Común, 2010 y DiNiece, 2010.

13 De un total de 789 establecimientos educativos de gestión privada que existen en la Ciudad de Buenos Aires, 441 reciben aportes estatales (ACIJ, 2011). Disponible en: http:// acij.org.ar/wp-content/uploads/2011/11/Subsidio-a-privadas-web.pdf 
Cuadro 2. Porcentaje de alumnos por calificación obtenida, según tipo de entorno sector de gestión de la escuela secundaria a la que asistieron

\begin{tabular}{|c|c|c|c|c|c|c|c|c|}
\hline \multirow{2}{*}{ Calificación } & \multicolumn{2}{|c|}{ NBı bajo } & \multicolumn{2}{|c|}{ NBI medio } & \multicolumn{2}{|c|}{$\begin{array}{c}\text { NBI alto y } \\
\text { asentamientos }\end{array}$} & \multicolumn{2}{|c|}{ Total } \\
\hline & $\begin{array}{l}\text { Sector } \\
\text { estatal }\end{array}$ & $\begin{array}{l}\text { Sector } \\
\text { privado }\end{array}$ & $\begin{array}{l}\text { Sector } \\
\text { estatal }\end{array}$ & $\begin{array}{l}\text { Sector } \\
\text { privado }\end{array}$ & $\begin{array}{l}\text { Sector } \\
\text { estatal }\end{array}$ & $\begin{array}{l}\text { Sector } \\
\text { privado }\end{array}$ & $\begin{array}{l}\text { Sector } \\
\text { estatal }\end{array}$ & $\begin{array}{l}\text { Sector } \\
\text { privado }\end{array}$ \\
\hline Aprobaron & 49 & 63 & 42 & 62 & 45 & 44 & 47 & 62 \\
\hline $\begin{array}{l}\text { Aprobaron } \\
\text { rendimiento alto }\end{array}$ & 34 & 46 & 29 & 44 & 33 & 32 & 33 & 45 \\
\hline $\begin{array}{l}\text { Aprobaron } \\
\text { rendimiento } \\
\text { medio }\end{array}$ & 15 & 17 & 13 & 18 & 12 & 12 & 14 & 17 \\
\hline No aprobaron & 11 & 11 & 10 & 11 & 11 & 13 & 11 & 11 \\
\hline Ausentes & 40 & 26 & 48 & 27 & 44 & 43 & 42 & 27 \\
\hline Total & $100 \%$ & $100 \%$ & $100 \%$ & $100 \%$ & $100 \%$ & $100 \%$ & $100 \%$ & $100 \%$ \\
\hline
\end{tabular}

Fuente: Elaboración propia con base en información proporcionada por el Departamento de Alumnos de Ciclo Básico Común, 2010 y DiNiece, 2010.

El análisis realizado permite identificar ciertas tendencias similares entre las categorías de NBI correspondientes al radio censal de la escuela, que no parecieran estar tan asociadas al sector de gestión de la escuela secundaria sino en mayor medida al nivel socioeconómico.

A continuación, se incorpora en el análisis el logro académico, presentando el análisis de la calificación obtenida por los alumnos ingresantes por sector de gestión y según las características socioeconómicas de las escuelas de nivel secundario de procedencia.

La probabilidad de aprobación de la materia Sociedad y Estado es mayor para aquellos alumnos provenientes de escuelas privadas: entre los alumnos que ingresaron en el primer cuatrimestre de 2010 y cursaron la materia seleccionada se observa que el $62 \%$ de los que asistieron a escuelas de gestión privada aprobaron la materia, mientras el $47 \%$ de los que estudiaron en escuelas de gestión estatal lograron hacerlo. Además, es mayor el porcentaje de alumnos con calificaciones altas, entre 7 y 10, entre quienes asistieron a escuelas de gestión privada (45\% frente a $33 \%$ de los que asistieron a escuelas estatales), aunque el porcentaje de desaprobación es similar entre ambos sectores de gestión ( $11 \%$ en cada caso). Finalmente, se registra un número muy elevado de alumnos que tienen ausente en su calificación final la última vez que cursaron la materia. Entre ellos, es menor el porcentaje de alumnos "ausentes" que asistieron a colegios privados $(27 \%)$ que el de quienes 
asistieron a escuelas secundarias estatales (42\%). Es decir que a lo largo del curso abandonaron la materia o no se presentaron el día de la evaluación un 15\% más de alumnos provenientes de escuelas públicas que de escuelas privadas.

Es decir que se verifica una diferencia de 15 puntos porcentuales entre los alumnos procedentes de escuelas estatales (frente a los que asistieron a secundarias privadas) que a lo largo del curso abandonaron la materia o no se presentaron el día de la evaluación.

En el gráfico 1 se resume el rendimiento obtenido con relación a las dos principales variables consideradas. El mismo permite analizar el modo en que el sector de gestión se asocia de manera diferencial a la aprobación en los distintos niveles de incidencia de NBI.

Se observa que quienes asistieron a escuelas ubicadas en radios censales de menor pobreza estructural son quienes logran mejores calificaciones, principalmente si la escuela de origen pertenecía al sector de gestión privada (46\%, frente al 34\% de gestión estatal).

Por su parte, entre los alumnos que concurrieron a escuelas localizadas en entornos de nivel medio, el 44\% de los que asistieron a secundarias privadas lograron calificaciones altas en la materia Sociedad y Estado, frente al 29\% de los que asistieron a secundarias estatales.

Por último, entre los estudiantes que cursaron sus estudios secundarios en escuelas ubicadas en entornos desfavorables, la incidencia de aquellos con rendimiento alto resulta independiente del sector de gestión de la escuela de origen.

Gráfico 1. Porcentaje de alumnos por calificación obtenida, según sector de gestión y tipo de entorno de la escuela secundaria a la cual asistieron

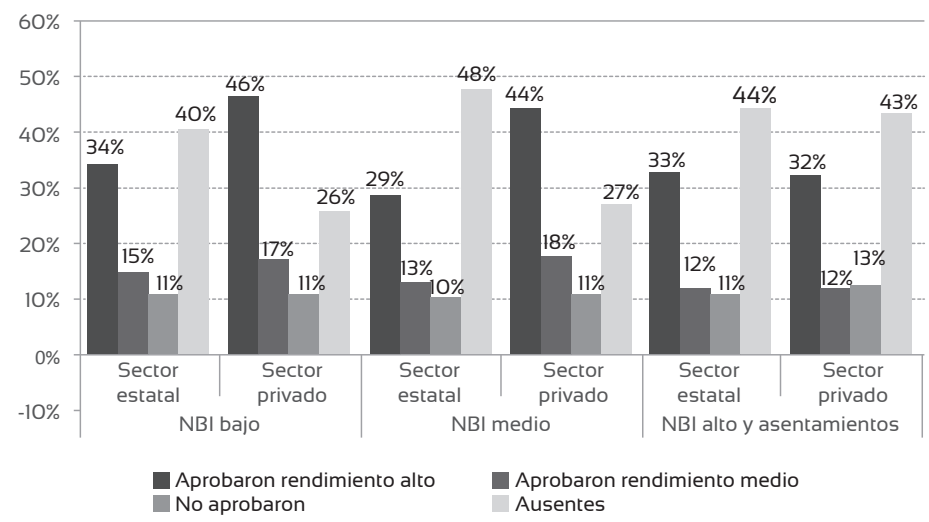

Fuente: Elaboración propia, con base en información proporcionada por el Departamento de Alumnos del Ciclo Básico Común, 2010 y DiNiece, 2010. 
Aprobación, sector de gestión y nivel socioeconómico de la escuela secundaria de origen

En los gráficos 2 a 4 se presenta la distribución de los alumnos que aprobaron sobre el total de cada atributo considerado (lo cual supone la combinación de las distintas variables analizadas conjuntamente con el sector de gestión de la escuela secundaria a la que asistieron y el NBI del entorno). Cabe aclarar que para hacer más robusto el análisis se considera exclusivamente a los alumnos que aprobaron, tanto aquellos con rendimiento medio (calificación entre 4 y 6) como aquellos con rendimiento alto (calificación de 7 o más).
Según los datos del gráfico 2, no se observan diferencias sustantivas en el logro de varones y mujeres de establecimientos secundarios de gestión estatal por un lado, y de gestión privada por el otro, ubicados en un mismo tipo de entorno social.

Sin embargo, entre quienes asistieron a escuelas ubicadas en los entornos más favorables (NBI bajo y medio), tanto las mujeres como los varones provenientes de secundarias privadas presentan un mayor porcentaje de aprobación. Estas diferencias privado/ estatal se reducen significativamente entre los alumnos que asistieron a escuelas ubicadas en contextos más vulnerables.

Con respecto a la edad, como se presenta en el gráfico 3, se advierte que entre los alumnos menores de 19

Gráfico 2. Porcentaje de alumnos que aprobaron por sexo, según sector de gestión y tipo de entorno de la escuela secundaria a la que asistieron (sobre el respectivo total de alumnos)
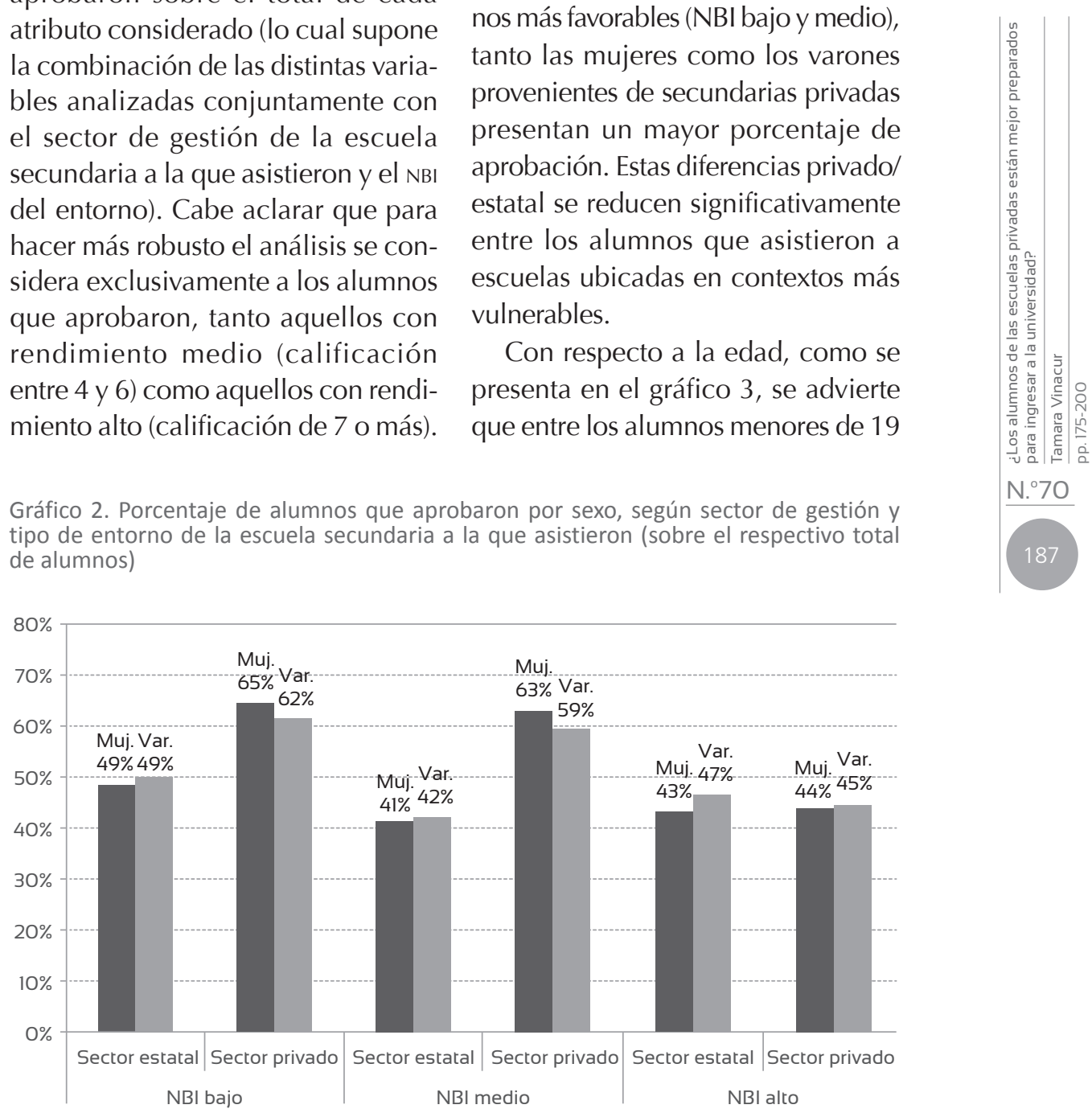

Fuente: Elaboración propia con base en información proporcionada por el Departamento de Alumnos del Ciclo Básico Común, 2010, y DiNiece, 2010. 
años existe un mayor porcentaje de aprobación que entre los de mayor edad. A su vez, se observa que los alumnos de menor edad que asistieron a escuelas secundarias de gestión privada ubicadas en contextos menos desfavorables presentan mayores porcentajes de aprobación (el $67 \%$ de los alumnos en radios censales con NBI bajo -el restante $33 \%$ de este grupo no aprobó o tiene calificación ausente- y $65 \%$ en radios censales con NBI medio) que los alumnos menores de 19 años que asistieron a escuelas emplazadas en radios censales similares pero de gestión estatal $(57 \%$ en radios censales con NBı bajo -43 \% no aprobó o tiene calificación ausente- y $48 \%$ en radios censales con NBI medio). Sin embargo, entre quienes asistieron a escuelas localizadas en entornos con alto nivel de pobreza estructural la asistencia a establecimientos de gestión estatal por parte de los menores de 19 años resulta más beneficiosa que la asistencia a establecimientos de gestión privada ( $56 \%$ y $49 \%$ respectivamente).

Por último, el gráfico 4 contempla el logro académico con relación al nivel educativo de la madre. Se advierte que independientemente del nivel de pobreza de la localización de la escuela secundaria y el sector de gestión, los alumnos que cuentan con madres con estudios universitarios completos presentan mayores tasas de aprobación.

Gráfico 3. Porcentaje de alumnos que aprobaron por rango de edad, según sector de gestión y tipo de entorno de la escuela secundaria a la que asistieron (sobre el respectivo total de alumnos)

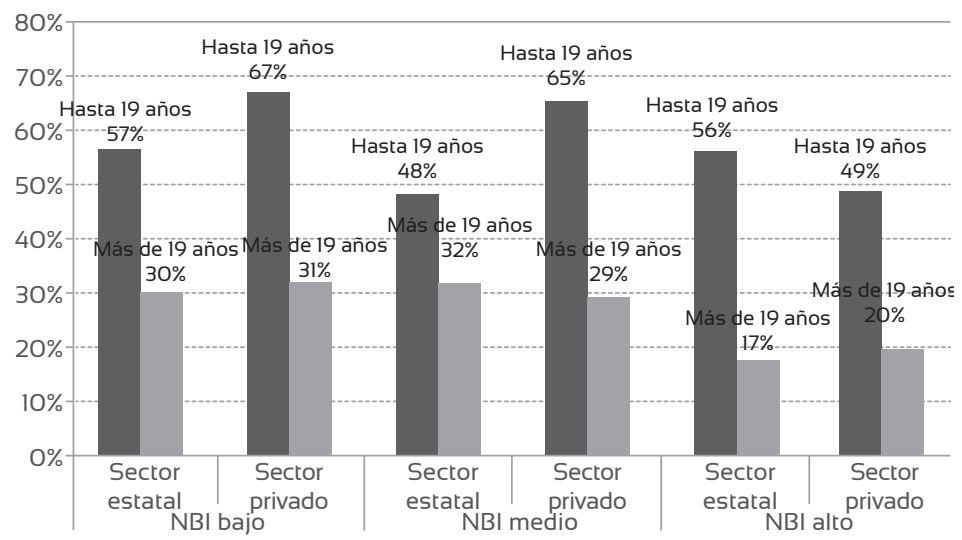

Fuente: Elaboración propia con base en información proporcionada por el Departamento de Alumnos del Ciclo Básico Común, 2010 y DiNiece, 2010. 
Gráfico 4. Porcentaje de alumnos que aprobaron por nivel de instrucción de la madre, según sector de gestión y tipo de entorno de la escuela secundaria a la que asistieron

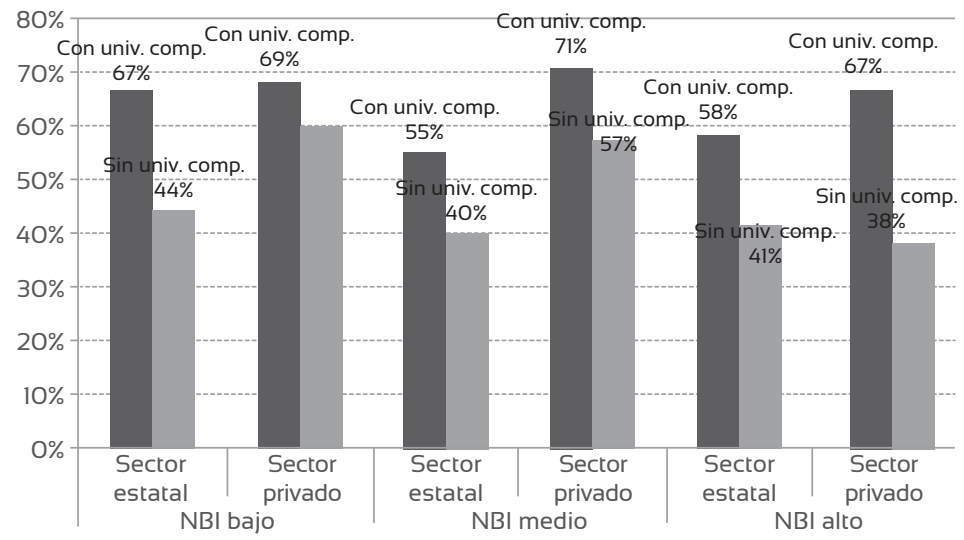

Fuente: Elaboración propia con base en información proporcionada por el Departamento de Alumnos del Ciclo Básico Común, 2010 y DiNiece, 2010.

Tiempo requerido para

la aprobación, sector

de gestión y nivel

socioeconómico de la

escuela secundaria de

\section{origen}

A continuación se analiza el tiempo requerido para aprobar la materia, considerando que del total de los ingresantes en 2010, un alto porcentaje lo hace dentro del primer año ${ }^{14}$. En este sentido, el análisis contempla el momento en que los alumnos aprueban la materia seleccionada, considerando las diferencias existen-

14 De acuerdo al Régimen de Promoción vigente, los alumnos cuentan con tres instancias para rendir el examen final, en caso de haber tenido un promedio mayor a 4 en los parciales e inferior a 7. 1. a instancia: fin del cuatrimestre en el que cursó la materia; 2. a instancia: inicio del siguiente cuatrimestre, posterior al que cursó la materia; y 3. ${ }^{a}$ instancia: fin del siguiente cuatrimestre, posterior al que cursó la materia (http:// www.cbc.uba.ar/). tes en función del sector de gestión y el radio censal donde estaba ubicada de la escuela secundaria a la que asistieron. Aun cuando el alumno puede aplazar hasta el fin del cuatrimestre posterior al que cursó la materia, se decidió contemplar una periodización mayor considerando la posibilidad de que se inscriba de nuevo como alumno repitente.

En primer lugar, como se mencionó, en el cuadro 3 se observa que los alumnos que aprueban la materia lo hacen fundamentalmente a lo largo del primer año posterior a haberla cursado. En segundo lugar, se identifica que los alumnos que asistieron a escuelas privadas logran aprobar la materia en un plazo menor. En tercer lugar, y de modo similar, aquellos alumnos cuyas escuelas están emplazadas en radios censales de NBI más bajo también logran aprobar antes. Resulta interesante analizar lo que 
ocurre con aquellos alumnos, tanto de gestión estatal como privada, que asistieron a escuelas secundarias ubicadas en un radio censal de alta incidencia de NBI. En ambos sectores de gestión, se encuentra que estos logran aprobar la materia en un plazo inferior a lo alcanzado por aquellos alumnos que asistieron a escuelas de radios censales medios.

Cuadro 3. Periodo de aprobación de la materia Sociedad y Estado (24), según sector de gestión y NBI del radio censal de la escuela secundaria a la que asistieron

\begin{tabular}{|c|c|c|c|c|c|c|c|c|}
\hline \multirow{2}{*}{ Periodo } & \multicolumn{4}{|c|}{ Sector estatal (\%) } & \multicolumn{4}{|c|}{ Sector privado (\%) } \\
\hline & NBI bajo & NBI medio & $\mathrm{NBI}$ alto & Asentamiento & NBI bajo & NBI medio & $\mathrm{NBI}$ alto & Asentamiento \\
\hline 2010-I & 43,2 & 38,2 & 42,2 & 40,8 & 45,0 & 44,5 & 42,3 & 41,2 \\
\hline 2010-II & 34,6 & 29,1 & 33,8 & 38,8 & 40,9 & 40,8 & 44,3 & 41,2 \\
\hline 2011-I & 9,8 & 12,7 & 15,7 & 10,2 & 8,3 & 8,3 & 6,2 & 5,9 \\
\hline 2011-II & 5,4 & 9,5 & 4,4 & 6,1 & 2,8 & 3,9 & 1 & 5,9 \\
\hline $2012-I$ & 3,5 & 5 & 1,5 & 2 & 1,6 & 1,3 & 4,1 & 2,9 \\
\hline 2012-II & 2,4 & 4,1 & 1,5 & 0 & 0,9 & 0,8 & 2,1 & 2,9 \\
\hline 2013-I - & 1,1 & 1,4 & 1 & 2 & 0,6 & 0,5 & 0 & 0 \\
\hline Total & $100 \%$ & $100 \%$ & $100 \%$ & $100 \%$ & $100 \%$ & $100 \%$ & $100 \%$ & $100 \%$ \\
\hline
\end{tabular}

\section{Conclusiones}

El artículo se centra en el análisis de los 8542 alumnos ingresantes al Ciclo Básico Común en 2010 y que aprobaron la materia Introducción al Estudio de la Sociedad y el Estado. Se presentan evidencias que permiten identificar que el sector de gestión de la escuela secundaria a la que asistieron y/o el porcentaje de NBI del radio censal donde la escuela está ubicada se encuentran asociados con las diferencias en las calificaciones obtenidas y el tiempo que necesitan los estudiantes para aprobar una de las materias comunes a todas las carreras.

El análisis realizado permite advertir, en primer lugar, que quienes llegan a la universidad son mayormente mujeres que asistieron a establecimientos educativos de nivel secundario (tanto de gestión estatal como privada) ubicados en radios censales con bajo NBI. Esto resulta consistente, por un lado, con la brecha de género que favorece a las mujeres en términos educativos, y por el otro, con la hipótesis de que existen procesos de selectividad 
que reproducen las desigualdades de origen y dificultan el acceso de los estudiantes de menores recursos. De hecho, hay ciertas condiciones sociales que se requieren para la aprobación y continuidad educativa, tales como la compra de materiales de estudio y traslado, por mencionar algunos, así como las necesidades materiales, tales como vivienda, salud, alimentación, etc. (Kisilevsky \& Veleda, 2002).

De manera coincidente, también son las mujeres, en particular aqueIlas que asistieron a establecimientos educativos de nivel privado, quienes aprueban en mayor proporción, en zonas de menor incidencia de NBI. Este hallazgo es consistente con la literatura internacional (Naylor \& Smith; 2004; Porto \& Di Gresia, 2001) y podría estar asociado a la materia seleccionada, ya que en algunos casos se identifican mejores rendimientos de mujeres en tareas de razonamiento verbal, comprensión lectora y establecimiento de relaciones lógicas (Hedges \& Nowell, 1995; Jacobs, 2002), aunque esto no se encuentra suficientemente justificado (McNabb et al., 2002).

Segundo, se observa un comportamiento diferencial entre quienes asistieron a escuelas ubicadas en zonas de menor incidencia de NBI, en donde hay una mayor tasa de aprobación entre quienes asistieron a escuelas privadas, a diferencia de lo que ocurre en escuelas localizadas en entornos con alto nivel de pobreza estructural, en donde la diferencia entre sectores se atenúa. Esto haría suponer que hay otros aspectos, probablemente vinculados con características actitudinales de los alumnos, tales como la persisten$\mathrm{cia}^{15}, \mathrm{u}$ otros factores pedagógicos e institucionales, derivadas del perfil de los docentes que trabajan en escuelas estatales en contextos más desfavorecidos, que podrían estar asociadas a un mejor rendimiento académico (Narodowski et al., 2014)

Tercero, se observa una diferencia en la edad de los alumnos ingresantes en función del sector de gestión de la escuela secundaria a la que asistieron. Los alumnos mayores de 19 años provienen en mayor proporción de escuelas de gestión estatal. También la edad pareciera estar asociada a una mayor aprobación, con independencia del radio censal y el sector de gestión de la escuela secundaria. Esto podría atribuirse a la relación que Chen y Kauffman (1997) establecen entre el desempeño académico y la trayectoria educativa anterior, particularmente en el caso de alumnos que pudieron haber repetido en el secundario o que por motivos económicos demoraron su ingreso a la universidad. De este modo, podría pensarse que los estudiantes de menor edad, con trayectorias educativas continuas, tienen mayores oportunidades en el ingreso a la universidad, ya que contarían con

15 La persistencia se refiere a la capacidad de un estudiante o su motivación para alcanzar sus propias metas académicas (Astin, 1975; Hagedorn, 2005). 
condiciones materiales y apoyos para poder lograrlo (Beltrán \& La Serna Studzinski, 2009; Guzmán, 2005 en Guzmán Gómez \& Serrano Sánchez, 2011).

Cuarto, se reconoce que entre los alumnos de escuelas privadas hay una mayor proporción de alumnos ingresantes con madres con nivel educativo universitario completo. De manera similar a lo discutido a nivel internacional, se identifica la influencia favorable del nivel educativo de la madre (Albert, 2000; Cea \& Mora, 1992; Di Gresia et al., 2005; Rahona, 2005).

Quinto, con relación al tiempo requerido para la aprobación se observa que quienes aprueban lo hacen fundamentalmente en el año posterior a cursar la materia. Esto resulta similar a lo que ocurre con quienes abandonan, que también, en promedio, lo hacen en el primer año (Aponte-Hernández, 2008), independientemente del sector de gestión de la escuela secundaria a donde hayan asistido. Es decir, son pocos los alumnos que al obtener una calificación baja como nota final de la materia presentan nuevamente el final o vuelven a cursar la materia luego del primer año. Bethencourt et al. (2008) plantean como hipótesis que los alumnos que obtienen bajas calificaciones se autoexcluyen porque consideran que no van a lograr revertir el resultado obtenido. También podría pensarse que conservan el ritmo de estudio de la secundaria, y que quizás están más motivados o focalizados en el estudio (Ferreyra, 2007) por lo que resulta más sencillo preparar la materia al haberla cursado hace poco tiempo.

Para finalizar, el análisis realizado permite identificar que los alumnos provenientes de escuelas secundarias privadas, en particular aquellas emplazadas en zonas de menor pobreza estructural, cuentan con mayores oportunidades para transitar exitosamente el primer año de universidad (tanto en la calificación obtenida como en el tiempo requerido para lograrlo), lo cual estaría reflejando una integración social y académica adecuada del alumno a la universidad (Pascarella \& Terenzini, 1991; Tinto, 1987, 2001). Sin embargo, sería preciso profundizar en las características de las escuelas de gestión estatal emplazadas en zonas de alto NBI que presentan un comportamiento diferencial con respecto a otras escuelas estatales, y permiten atenuar las diferencias entre sectores de gestión.

Las altas tasas de abandono que presenta el СвC (Plotno, 2009), con independencia del sector de gestión y radio censal de la escuela secundaria, ponen en evidencia que resulta necesario complementar el análisis a partir de la consideración de factores institucionales y pedagógicos. Esto permitiría reconocer que el 
logro académico de los alumnos es producto de un complejo inter-juego entre sus características personales, las estrategias de enseñanza y un contexto institucional más amplio (Amago, 2004; Biggs 2003; Ezcurra, 2009; Meyer \& Land 2006; Prosser \& Trigwell 1999).

\section{Referencias}

Acuña, C. \& Chudnovsky, M. (2002). El sistema de salud en Argentina. Documento de trabajo 60. Centro de Estudios para el DesarroIlo Institucional, CEDI. Fundación Gobierno.

Alava, S. (1999). Médiation(s) et métier d'étudiant. Bulletin des Bibliotheques de France, 44 (1), 8-15.

Albert, C. (2000). Higher education demand in Spain: the influence of labour market signals and family background, Higher Education, 40 (2): 147-162.

Altbach, P; Reisberg, L. \& Rumbley L. (2009, July) Trends in global higher education: Tracking an academia revolution. Informe preparado para la Unesco 2009 World Conference on Higher Education, Paris.

Amago, L. (2004). Principales dificultades de los estudiantes de primer ingreso al grado. Estudio preliminar sobre el Estado del Conocimiento. Secretaría Académica; Mimeo/ UNGS, Buenos Aires.
Aponte-Hernández, E. (2008). Desigualdad, inclusión y equidad en la educación superior en América Latina y el Caribe: Tendencias y escenario alternativo en el horizonte 2021. enTendencias de la Educación Superior en América Latina y el Caribe (pp. 113-154). Unesco.

Astin, A. W. (1975). Preventing students from dropping out. San Francisco: The Jossey-Bass Series in Higher Education

Beltrán, A. \& Laserna Studzinski, K. (2009). ¿Qué explica la evolución del rendimiento académico universitario? Un estudio de caso en la Universidad del Pacífico. Disponible en http:// disde.minedu.gob.pe/xmlui/ handle/123456789/1513.

Bethencourt, J. T.; Cabrera, L.; Hernández, J. A.; Álvarez, P. \& González, M. (2008). Variables psicológicas y educativas en el abandono universitario. Revista Electrónica de Investigación Socioeducativa, 16 (6), 603-622.

Biggs, J. (2003). Teaching for quality learning at university ( $2^{\text {nd }}$. ed.). Buckingham: Society for Research into Higher Education, Open University Press.

Birch, E. \& Miller, P. (2007). The influence of type of high school attended on university performance. Australian Economic Papers. 46 (1), 1-17. 
Bishop, J. (1989). Is the test score decline responsible for the productivity grow decline? American Economic Review, 75 (3), 1016-30.

Bishop, J. (1992). The impact of academic competencies on wages, unemployment, and job performance. En Carnegie-Rochester Conference Series on Public Policy, 37, 127-194.

Boulet, P. (2005). La Universidad y los otros. Revista Iberoamericana de educación. 36 (10), 1-12. Disponible en: http://www. rieoei.org/deloslectores/1017Boulet.pdf

Bourdieu, P. \& Passeron, J. C. (1964). Los herederos. Estudiantes y la cultura. Buenos Aires: Siglo xxı.

Brunner, J. \& Elacqua, G. (2004). Factores que inciden en una educación efectiva. Evidencia internacional. Revista Virtual La educación, 48-49 (139-140), Organización de Estados Americanos.

Camilloni, A. (2009). Los desafíos del ingreso a la universidad. Entrevista a Alicia R. Wigdorovitz de Camilloni. En S. Gvirtz, \& A. Camou, (coord.). La universidad argentina en discusión (pp. 279-297). Buenos Aires: Editorial Granica.

Cea, F. \& Mora, J. G. (1992). Análisis socioeconómico de la elección de estudios superiores. Estadística Española, 34 (129), 61-92.

Chen, x. \& Kauffman, P. (1997). Risk and resilience. The effects of dropping out of school. Ponencia presentada durante la reunión anual de la American Association of Education Research. Chicago, Illinois.

Cols, E. (2005). Taller de Ciencias Fácticas: Evaluación de la Experiencia Piloto. Secretaría Académica, Universidad Nacional General Sarmiento.

Cols. E. (2007). El primer año del grado: Estrategias y dispositivos de apoyo y mejora. Informe final. Secretaría Académica, Universidad Nacional General Sarmiento.

Correa J. J. (2004). Determinantes del rendimiento educativo de los estudiantes de secundaria en Cali: un análisis multinivel. Sociedad y Economía. Disponible en http://www.redalyc.org/ articulo.oa?id=99617648003.

Coulon, A. (1997). Le métier d'étudiant: L'entrée dans la vie universitaire. París: Económica-Anthropos.

Crissman, J. \& Upcraft, M. L. (2005). The keys to first-year student persistence. En M. Lee Upcraft, Jennifer Gardner \& Betsy Barefoot (eds.). Challenging and Supporting the First-Year Student. A handbook for improving the first year of college (pp. 27-46). San Francisco, Jossey-Bass. 
DiGresia, L.; Fazio, M. V.; Porto, A. \& Ripani, L. (2005). Rendimiento y productividad de los estudiantes. El caso de las universidades públicas argentinas. En A. Porto, (editor), Economía de la Educación Universitaria: ArgentinaBrasil-Perú, (pp. 19-125).

Di Gresia, L.; Porto, A. \& Ripani, L. (2002). Rendimiento de los estudiantes de las universidades públicas argentinas. Universidad Nacional de la Plata. Departamento de Economía. Documento de trabajo 45. Disponible en: www.depeco.econo.unlp.edu. ar/doctrab/doc45.pdf

Entwistle, N. (2000). Promoting deep learning through teaching and assessment: conceptual frameworks and educational contexts. TLRP Conference, Leicester.

Ezcurra, A. M. (2003). Principales dificultades de los alumnos de primer ingreso al grado (Primer Ciclo Universitario). Universidad Nacional General Sarmiento.

Ezcurra, A. M. (2005). Diagnóstico preliminar de las dificultades de los alumnos de primer ingreso a la educación superior. Perfiles Educativos, 27-107.

Ezcurra, A. M. (2007). Los estudiantes de nuevo ingreso. Democratización y responsabilidad de las instituciones universitarias. Cuadernos de Pedagogía Universitaria, 2. ProRectoría de Graduación, Universidad de San Pablo, San Pablo, Brasil.
Ezcurra, A. (2009). Educación universitaria, una inclusión excluyente. Conferencia inaugural, Tercer Encuentro Nacional sobre Ingreso Universitario. Organizado por la Universidad Nacional de Río Cuarto. http:// www.ideas. org.ar/empezar-descargamiento/foro-de-educaciónsuperior/6-educación-universitaria-una-inclusión-excluyente.

Ezcurra, A. M (2011). Igualdad en educación superior. Un desafío mundial. Los Polvorines. Universidad Nacional General Sarmiento - IEC/CONADU. 108.

Ferreyra, M. G. (2007). Determinantes del desempeño universitario: efectos heterogéneos en un modelo censurado. Tesis de Maestría en Economía. Universidad Nacional de La Plata.

Filmus, D.; Miranda, A. y Otero, A. (2004). La construcción de trayectorias laborales entre los jóvenes egresados de la escuela secundaria. En Jacinto, C. (coord.) ¿Educar para qué trabajo? Discutiendo rumbos en América Latina (pp. 201-222). Buenos Aires: redEtis (IIPE-IDES)/ MTEY SS/MECYT/La Crujía.

Francis, D.; Shaywitz, S.; Stuebing, K.; Shaywitz, B. \& Fletcher, J. (1994). The measurement of change: Assessing behavior over time and within developmental context. En G. R. Lyon (Comp.), Frames of reference: Measurement of learning disabilities. Baltimore, MD: Paul Books. 
García de Fanelli, A. M. (2005). Acceso, abandono y graduación en la educación superior argentina. Sistema de Información de Tendencias Educativas en América Latina. UnesCo-IIPE-OEI Garcia Guadilla, C. (2006). Modelos de acceso y políticas de ingreso. En Conocimiento, educación superior y sociedad en América Latina. Caracas: Nueva Sociedad.

García Jiménez, M. V.; Alvarado Izquierdo J. M. \& Jiménez Blanco, A. (2000). La predicción del rendimiento académico: regresión lineal versus regresión logística. Psicothema, 12 (2), 248-252. Disponible en: http://redalyc.uaemex.mx/redalyc/ pdf/727/72797059.pdf

Gessaghi V. \& P. Llinás (2005). Democratizar el acceso a la educación superior. En Documentos de trabajo. Centro de Implementación de Políticas Públicas para la Equidad y el Crecimiento (Cippec).

Guzmán Gómez \& Serrano Sánchez. (2011). Las puertas del ingreso a la educación superior. Revista de la Educacion Superior, 40 (157), 31-53.

Hagedorn, L. S. (2005). How to define retention: A new look at an old problem. En A. Seidman (Ed.), College student retention (pp. 89-105). Westport: Praeger Publishers.

Hedges, L. V. \& Nowell, A. (1995). Sex differences in mental test scores. Variability, and numbers of high-scoring individuals. Science, 269, 41-45

Hernández-González, R.; Vadillo-Bueno, G. \& Rivera-Leonides, S. (2008). Eficacia educativa: avances de un modelo para la educación superior. Magis, Revista Internacional de Investigación en Educación, 1(1), 63-80. Pontificia Universidad Javeriana. Disponible en: http://www.redalyc.org/ pdf/2810/281021687005.pdf

Jacobs G. (2002). Non academic factors affecting the academic success of Grenadian students at St. Georges University. SGU; 120-33.

Jansen, E. \& Shure J. M. (2010). The effect of secondary school skills preparation on first year university achievement. Educational Studies, 36 (5), 569-580.

Kisilevsky, M. \& Veleda, C. (2002). Dos estudios sobre el acceso a la educación superior en la Argentina. Buenos Aires: IIPE-Unesco.

Ladrón de Guevara, C. (2000). Condiciones sociales y familiares y fracaso escolar. En: A. Marchesi, \& C. Hernández Gil, (eds.) El fracaso escolar. Madrid: Doce Calles. 
McDonough, P. (1997). Choosing colleges: How social class and schools structure opportunity. Nueva York: State University of New York Press.

McKenzie, K. \& Schweitzer, R. (2001). Who succeeds at university? Factor predicting academic performance in first year Australian University students. Higher Education Research and Development, 20 (1).

McNabb, R.; Pal, S. \& Sloane, P. (2002). Gender differences in educational attainment: The case of university students in England and Wales. Economica, 69 (275), 481-503.

Meyer, J. H. F. \& Land, R. (2006). Threshold concepts and troublesome knowledge: Issues of liminality. En: J. H. F. Meyer, y R. Land (eds.), Overcoming barriers to student understanding: Threshold concepts and troublesome knowledge. Londres y Nueva York: Routledge, pp. 19-32.

Narodowski, M. \& Andrada, M. (2000). Segregación socioeconómica y regulaciones en el sistema educativo argentino. Documento 36. Buenos Aires: Centro de Estudios para el Desarrollo Institucional (CEDI).

Narodowski, M.; Vinacur, T. \& Alegre S. (2014). Los mejores maestros. Mitos, leyendas y realidades. Buenos Aires: Prometeo.
Naylor, R. A \& Smith, J. (2004). Determinants of educational success in higher education. En G. Johnes \& J. Johnes (eds.) International Handbook in the Economics of Education. Elgart.

Pascarella, E. T. \& Terenzini, P. T. (1991). How college affects students. San Francisco: Jossey-Bass. Plotno, G. (2011). Movilidad educativa, reclutamiento universitario y educación familiar. Instituto de Investigaciones Gino Germani. Disponible en: http:// seminariosms.fahce.unlp.edu. ar/sceyms/sceyms-2011/actas/ RC_Plotno.pdf

Ponsot, B. E., Varela, L.; Sinha, S. \& Valera, J. (2009). Un modelo de regresión logística del rendimiento en los estudios universitarios: Caso Faces-ULA. Actualidad Contable Faces, 12 (18), 81-102. Disponible en: http://redalyc.uaemex. $\mathrm{mx} / \mathrm{src} / \mathrm{inicio} / \mathrm{ArtPdfRed}$. jsp?iCve $=25712300008$

Porcel, E.; Dapozo, G. \& López, M. (2010). Predicción del rendimiento académico de alumnos de primer año de la Facena (UNNE) en función de su caracterización socioeducativa. Revista Electrónica de Investigación Educativa, 12 (2). Disponible en: http://redie.uabc.mx/vol12no2/ contenido-porcel dapozo.html 
Porto, A. \& Di Gresia L. (2001). Rendimiento de estudiantes universitarios y sus determinantes. Departamento de Economía: UNLP.

Prosser, M. \& Trigwell, K. (1999). Relational Perspectives on Higher Education Teaching and Learning in the Sciences. Studies in Science Education, 33, 31-60.

Rahona, M. (2005). La influencia del entorno socioeconómico en la realización de estudios universitarios: una aproximación al caso español en la década de los noventa. Documento de trabajo 215. Fundación de las Cajas de Ahorros.

Rinaudo, M. C.; Chiecher, A. \& Donolo, D. (2003). Motivación y uso de estrategias en estudiantes universitarios. Su evaluación a partir del Motivated Strategies Learning Questionnaire. Anales de Psicología, 19 (1) 107-119.

Sirota, R. (2000). El 'oficio de alumno' y la sociología. En G. Frigerio, M. Poggi y M. Giannoni: Políticas, instituciones y actores en educación. Buenos Aires: Coedición Novedades Educativas, Centro de Estudios Multidisciplinarios.

Teobaldo, M. (1996). Evaluación de la calidad educativa en el primer año universitario. La Universidad Ahora, 10.

Teobaldo, M. (2002). El aprendizaje del oficio de alumno en el primer año de la universidad: concepciones previas sobre aprender y enseñar. Contextos institucionales y familiares. I Congreso Internacional y II Nacional: La Educación Frente a los Desafíos del Tercer Milenio: Camino hacia la Libertad. Unión de Educadores de Provincia de Córdoba y Escuela Normal Superior Dr. Alejandro Carbó.

Tinto, V. (1987). Leaving College. Chicago: The University of Chicago Press.

Tinto, V. (2001). Rethinking the first year of college. Higher Education Syracuse University: Monograph Series.

Trow, M. (2005). Reflections on the Transitions from Elite to Mass to Universal Access: Forms of Higher Education in Modern Societies since WWII. En eScholarship Repository, University of California. Disponible en: http://repositories.cdlib.org/igs/ WP2005-4

Unesco (2002). Declaración de la Conferencia Mundial sobre Educación Superior, 5 al 9 de octubre de 1998, en la Sede de Unesco, París. Perspectivas de la Educación Superior en el siglo XXI, CRUE, Madrid. 
Valdivieso, M.; Monar, K. \& Granda, M. (2004). Análisis de los determinantes del rendimiento de los estudiantes de Espol 2002. Revista Tecnológica, 17(1), 213-218.

Wingate, U. (2007, July). A framework for transition supporting "learning to learn" in higher education. Higher Education Quaterly, 61 (3), 391-405. 


\section{Anexo}

Cuadro 4. Características demográficas de los alumnos ingresantes en 2010, según sector de gestión y NBI del radio censal de la escuela secundaria a la que asistieron

\begin{tabular}{|c|c|c|c|c|c|c|c|c|}
\hline \multirow[b]{2}{*}{$\begin{array}{c}\text { Características } \\
\text { sociodemográficas }\end{array}$} & \multicolumn{4}{|c|}{ Escuela secundaria estatal (\%) } & \multicolumn{4}{|c|}{ Escuela secundaria privada (\%) } \\
\hline & $\begin{array}{l}\mathrm{NBI} \\
\text { bajo }\end{array}$ & $\begin{array}{c}\mathrm{NBI} \\
\text { medio }\end{array}$ & $\begin{array}{l}\mathrm{NBI} \\
\text { alto }\end{array}$ & Asentamiento & $\begin{array}{l}\mathrm{NBI} \\
\text { bajo }\end{array}$ & $\begin{array}{l}\mathrm{NBI} \\
\text { medio }\end{array}$ & $\begin{array}{l}\text { NBI } \\
\text { alto }\end{array}$ & Asentamiento \\
\hline $\begin{array}{l}\text { Total de ingresantes al } \\
\text { CBC/ Tipo de escuela }\end{array}$ & 69 & 15 & 13 & 3 & 74 & 20 & 4 & 2 \\
\hline Mujer & 41 & 8 & 7 & 1 & 45 & 13 & 2 & 2 \\
\hline Varón & 29 & 7 & 6 & 2 & 29 & 7 & 2 & 1 \\
\hline Hasta 19 años & 49 & 9 & 9 & 2 & 67 & 18 & 3 & 2 \\
\hline Más de 19 años & 20 & 6 & 4 & 1 & 7 & 2 & 1 & 1 \\
\hline Sin hijos & 65 & 13 & 12 & 3 & 73 & 20 & 4 & 2 \\
\hline Con hijos & 4 & 1 & 1 & 0 & 1 & 0 & 0 & . \\
\hline Argentino & 65 & 13 & 12 & 3 & $73 \%$ & $20 \%$ & $4 \%$ & $2 \%$ \\
\hline De $A L C$ & 4 & 1 & 1 & 0 & 1 & 0 & 0 & 0 \\
\hline De otro país & 0 & . & 0 & . & 1 & 0 & . & . \\
\hline $\begin{array}{l}\text { Padre con } \\
\text { universitario completo }\end{array}$ & 15 & 2 & 3 & 0 & 31 & 7 & 1 & 0 \\
\hline $\begin{array}{l}\text { Padre sin universitario } \\
\text { completo }\end{array}$ & 55 & 13 & 10 & 3 & 43 & 14 & 2 & 2 \\
\hline $\begin{array}{l}\text { Madre con } \\
\text { universitario completo }\end{array}$ & 14 & 2 & 3 & 0 & 29 & 7 & 1 & 0 \\
\hline $\begin{array}{l}\text { Madre sin } \\
\text { universitario completo }\end{array}$ & 55 & 13 & 10 & 3 & 45 & 13 & 2 & 2 \\
\hline $\begin{array}{l}\text { Consultorio, hospital } \\
\text { privado o prepagada }\end{array}$ & 12 & 2 & 2 & 0 & 25 & 6 & 1 & 0 \\
\hline Obra social & 40 & 8 & 7 & 2 & 44 & 12 & 2 & 1 \\
\hline $\begin{array}{l}\text { Hospital público u } \\
\text { otro }\end{array}$ & 18 & 6 & 4 & 1 & 6 & 2 & 0 & 1 \\
\hline
\end{tabular}

Fuente: Elaboración propia con base en información proporcionada por el Departamento de Alumnos del Ciclo Básico Común, 2010 y DiNiece, 2010. 